\title{
GENERATORS OF FINITE FIELDS WITH PRESCRIBED TRACES
}

\author{
LUCAS REIS and SÁVIO RIBAS
}

(May 10, 2021)

\begin{abstract}
This paper explores the existence and distribution of primitive elements in finite field extensions with prescribed traces in several intermediate field extensions. Our main result provides an inequality-like condition to ensure the existence of such elements. We then derive concrete existence results for a special class of intermediate extensions.
\end{abstract}

Keywords and phrases: finite fields; primitive elements; field trace; character sums. Mathematics Subject Classification (2010): 12E20 (primary); 11T24 (secondary).

\section{Introduction}

Given a prime power $q$ and $n>1$ a positive integer, let $\mathbb{F}_{q}$ be the finite field with $q$ elements and $\mathbb{F}_{q^{n}}$ the unique $n$-degree field extension of $\mathbb{F}_{q}$. The intermediate extensions of $\mathbb{F}_{q^{n}}$ over $\mathbb{F}_{q}$ are exactly the finite fields $\mathbb{F}_{q^{d}}$ with $d$ a divisor of $n$. It is well-known that the multiplicative group $\mathbb{F}_{q^{n}}^{*}$ is cyclic; any generator of such group is called primitive. Primitive elements play important roles in a wide variety of applications in Cryptography and, perhaps, the most notably is the Diffie-Hellman key exchange [4]. Primitive elements with further specified properties have been extensively studied in the past few decades. The motivation comes from both theoretical and practical matters.

For instance, the celebrated Primitive Normal Basis Theorem states that, for any $n \geq 1$ and any prime power $q$, there exists a primitive element $\alpha \in \mathbb{F}_{q^{n}}$ such that $\alpha$ is normal over $\mathbb{F}_{q}$, i.e., the set $\left\{\alpha, \alpha^{q}, \ldots, \alpha^{q^{n-1}}\right\}$

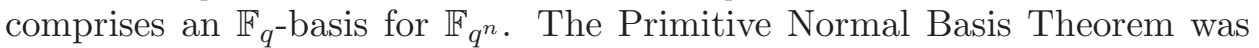
proved by Lenstra and Schoof [6] and a proof without any use of computers was later given by Cohen and Huczynska [2]. Cohen [1] also explored the

(C) XXXX Australian Mathematical Society 0263-6115/XX $\$$ A2.00+0.00 
existence of primitive elements in $\mathbb{F}_{q^{n}}$ with prescribed trace $a \in \mathbb{F}_{q}$, i.e., primitive elements $\alpha \in \mathbb{F}_{q^{n}}$ such that

$$
\operatorname{Tr}_{q^{n} / q}(\alpha)=\sum_{i=0}^{n-1} \alpha^{q^{i}}=a
$$

He has shown that, up to genuine exceptions, it is possible to find primitive elements in $\mathbb{F}_{q^{n}}$ satisfying equation (1). More specifically, we have the following result.

TheOREM 1.1. Let $q$ be a prime power, $n$ a positive integer and $a \in \mathbb{F}_{q}$. Then there exists a primitive element $\alpha \in \mathbb{F}_{q^{n}}$ such that $\operatorname{Tr}_{q^{n} / q}(\alpha)=$ a unless $a=0$ and $n=2$ or $a=0, n=3$ and $q=4$.

In this paper, we discuss the existence of primitive elements in $\mathbb{F}_{q^{n}}$ with prescribed traces in several intermediate extensions $\mathbb{F}_{q^{d}}$ of $\mathbb{F}_{q^{n}}$. In other words, given $n>1, d_{1}<\ldots<d_{k}<n$ divisors of $n$ and $a_{j} \in \mathbb{F}_{q} d_{i}$, we discuss the existence of a primitive element $\alpha \in \mathbb{F}_{q^{n}}$ such that, for each $1 \leq j \leq k$, we have that

$$
\operatorname{Tr}_{q^{n} / q^{d_{j}}}(\alpha)=\sum_{i=0}^{n / d_{j}-1} \alpha^{i q^{i d_{j}}}=a_{j} .
$$

Our main result, Theorem 2.3, provides an inequality-like condition to ensure the existence of such elements, that might easily yield asymptotic existence results. We then present a special instance where we can obtain effective results. In particular we prove that, up to few critical cases, there exists a primitive element $\alpha \in \mathbb{F}_{q^{n}}$ with arbitrary prescribed traces in any

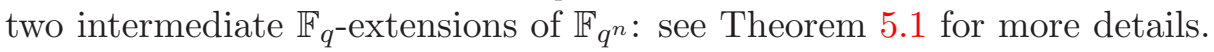

The structure of the paper is as follows. In Section 2 we introduce some useful notation and present our main result. Section 3 provides background material that is used along the way and some auxiliary results. In Section 4 we prove our main result. Finally, in Section 5, we restrict our problem to a special class of intermediate extensions, where our results are sharpened.

\section{Main Results}

Before we state our main result, we introduce some notation and discuss on a natural condition that we have to impose in the problem. Throughout this paper, $q$ is a prime power and $\mathbb{F}_{q}$ is the finite field with $q$ elements.

Definition 2.1. For $n>1, d$ a divisor of $n$ and $\alpha \in \mathbb{F}_{q^{n}}$, we set

$$
\operatorname{Tr}_{n / d}(\alpha)=\sum_{i=0}^{n / d-1} \alpha^{q^{d i}}
$$


the trace of $\alpha$ over $\mathbb{F}_{q^{d}}$.

Recall that the trace is transitive, i.e., if $e$ divides $d$ and $d$ divides $n$, then for any $\alpha \in \mathbb{F}_{q^{n}}$ we have that $\operatorname{Tr}_{n / e}(\alpha)=\operatorname{Tr}_{n / d}\left(\operatorname{Tr}_{d / e}(\alpha)\right)$. In particular, if $d_{1}<\ldots<d_{k}<n$ are divisors of $n$ and we pick $a_{i} \in \mathbb{F}_{q^{d_{i}}}, 1 \leq i \leq k$, then the existence of an element $\alpha \in \mathbb{F}_{q^{n}}$ with $\operatorname{Tr}_{n / d_{i}}(\alpha)=a_{i}$ is necessarily conditional on the following identities

$$
\operatorname{Tr}_{d_{i} / \operatorname{gcd}\left(d_{i}, d_{j}\right)}\left(a_{i}\right)=\operatorname{Tr}_{n / \operatorname{gcd}\left(d_{i}, d_{j}\right)}(\alpha)=\operatorname{Tr}_{d_{j} / \operatorname{gcd}\left(d_{i}, d_{j}\right)}\left(a_{j}\right), 1 \leq i, j \leq k .
$$

REMARK. As recently shown by the first author in [8], the equations in (2) are also sufficient and, in this case, there exist exactly $q^{n-\lambda}$ elements in $\mathbb{F}_{q^{n}}$ with $\operatorname{Tr}_{n / d_{i}}(\alpha)=a_{i}$ for $1 \leq i \leq k$, where

$$
\begin{aligned}
\lambda & =\operatorname{deg}\left(\operatorname{lcm}\left(x^{d_{1}}-1, \ldots, x^{d_{k}}-1\right)\right) \\
& =d_{1}+\ldots+d_{k}+\sum_{i=2}^{k}(-1)^{i+1} \sum_{1 \leq \ell_{1}<\cdots<\ell_{i} \leq k} \operatorname{gcd}\left(d_{\ell_{1}}, \ldots, d_{\ell_{i}}\right) .
\end{aligned}
$$

The proof of this result is a simple application of the Chinese Remainder Theorem for the ring $\mathbb{F}_{q}[x]$. For more details, see Theorem 4.1 in [8].

The equations in (2) imply that if $d_{i}$ divides some $d_{j}$, then the equality $\operatorname{Tr}_{n / d_{i}}(\alpha)=a_{i}$ is already implied by $\operatorname{Tr}_{n / d_{j}}(\alpha)=a_{j}$. So we may restrict ourselves to divisors $d_{1}<\ldots<d_{k}$ of $n$ such that $d_{i} \nmid d_{j}$ for any $1 \leq i<$ $j \leq k$. In addition, the case $k=1$ is completely settled by Cohen [1], so we assume that $k>1$, i.e., $n$ is not a prime power. We introduce a useful notation.

DeFinition 2.2. Let $n>1$ be an integer that is not a prime power and $1<k<\sigma_{0}(n)$, where $\sigma_{0}(n)$ denotes the number of positive divisors of $n$.

(i) $\Lambda_{k}(n)$ stands for the set of $k$-tuples $\mathbf{d}=\left(d_{1}, \ldots, d_{k}\right)$, where $d_{1}<\ldots<$ $d_{k}<n$ are divisors of $n$ such that $d_{i}$ does not divide $d_{j}$ for every $1 \leq i, j \leq k$ with $i \neq j$.

(ii) For $\mathbf{d}=\left(d_{1}, \ldots, d_{k}\right) \in \Lambda_{k}(n)$, set $\mathbb{F}(\mathbf{d})=\prod_{i=1}^{k} \mathbb{F}_{q^{d_{i}}}$ and

$$
\lambda(\mathbf{d})=d_{1}+\ldots+d_{k}+\sum_{i=2}^{k}(-1)^{i+1} \sum_{1 \leq \ell_{1}<\cdots<\ell_{i} \leq k} \operatorname{gcd}\left(d_{\ell_{1}}, \ldots, d_{\ell_{i}}\right) .
$$

Moreover, for $\mathbf{d}=\left(d_{1}, \ldots, d_{k}\right) \in \Lambda_{k}(n)$ and $\mathbf{a}=\left(a_{1}, \ldots, a_{k}\right) \in \mathbb{F}(\mathbf{d})$, the $k$-tuple $\mathbf{a}$ is $\mathbf{d}$-admissible if, for any $1 \leq i<j \leq k$, we have that

$$
\operatorname{Tr}_{d_{i} / \operatorname{gcd}\left(d_{i}, d_{j}\right)}\left(a_{i}\right)=\operatorname{Tr}_{d_{j} / \operatorname{gcd}\left(d_{i}, d_{j}\right)}\left(a_{j}\right) .
$$


From previous observation, we only need to consider $\mathbf{d}$-admissible $k$ tuples. Our main result can be stated as follows.

TheOREM 2.3. Let $n>1$ be an integer that is not a prime power, $1<$ $k<\sigma_{0}(n), \mathbf{d}=\left(d_{1}, \ldots, d_{k}\right) \in \Lambda_{k}(n)$, and $\mathbf{a}=\left(a_{1}, \ldots, a_{k}\right) \in \mathbb{F}(\mathbf{d}) a \mathbf{d}-$ admissible $k$-tuple. Then there exists a primitive element $\alpha \in \mathbb{F}_{q^{n}}$ with prescribed traces $\operatorname{Tr}_{n / d_{i}}(\alpha)=a_{i}$ for every $1 \leq i \leq k$ provided that

$$
q^{n / 2-\lambda(\mathbf{d})} \geq W\left(q^{n}-1\right)
$$

where $W(t)$ denotes the number of squarefree divisors of $t$.

In the context of Theorem 2.3, we also obtain the following minor result.

TheOREM 2.4. We have that Theorem 2.3 also holds if the condition $q^{n / 2-\lambda(\mathbf{d})} \geq W\left(q^{n}-1\right)$ is replaced by the inequality $\operatorname{lcm}\left(d_{1}, \ldots, d_{k}\right)<n$.

While the proof of Theorem 2.4 is a straightforward combination of Theorem 4.1 in [8] and Theorem 1.1, the proof of Theorem 2.3 relies on character sum methods to count elements in finite fields with specified properties. We follow the traditional approach that is presented in $[1,2,6]$. In this approach, we frequently need to simplify character sums by detecting trivial Gauss sums; in our case, we employ a result from [8] concerning special zero sums in finite fields.

\section{Preliminaries}

This section provides background material that is used throughout the paper and some auxiliary results.

3.1. Characters and characteristic functions Here we provide character sum expressions for the characteristic functions of elements in finite fields with specified properties. We start by recalling some basics on characters over finite fields.

Fix $\alpha \in \mathbb{F}_{q^{n}}$ a primitive element. A typical multiplicative character of $\mathbb{F}_{q^{n}}$ is a function $\eta: \mathbb{F}_{q^{n}}^{*} \rightarrow \mathbb{C}$ given by $\eta\left(\alpha^{k}\right)=e^{\frac{2 \pi i k t}{q^{n}-1}}$ for some positive integer $t \leq q^{n}-1$. The character $\eta_{1} \equiv 1$ is the trivial multiplicative character. The set of multiplicative characters of $\mathbb{F}_{q^{n}}$ form a (multiplicative) cyclic group of order $q^{n}-1$. In particular, for each divisor $t$ of $q^{n}-1$, there exist exactly $\varphi(t)$ multiplicative characters of order $t$; we denote the set of such characters by $\Gamma(t)$. We extend the evaluation of multiplicative characters to the element $0 \in \mathbb{F}_{q^{n}}$ by letting $\eta(0)=0$.

If $p$ is the characteristic of $\mathbb{F}_{q}$, say $q=p^{s}$, and $m$ is any divisor of $n$, the canonical additive character of $\mathbb{F}_{q^{m}}$ is the function $\chi: \mathbb{F}_{q^{m}} \rightarrow \mathbb{C}$ given by

$$
\chi(\beta)=e^{\frac{2 \pi i \mathcal{T}_{m}(\beta)}{p}},
$$


where $\mathcal{T}_{m}(\beta)=\sum_{i=1}^{m s-1} \beta^{p^{i}} \in \mathbb{F}_{p}$ is the absolute trace function from $\mathbb{F}_{q^{m}}$ to $\mathbb{F}_{p}$. For each $c \in \mathbb{F}_{q^{m}}$, we set $\chi_{c}(\beta)=\chi(c \cdot \beta)$, which is another additive character of $\mathbb{F}_{q^{m}}$. In fact, the set of additive characters of $\mathbb{F}_{q^{m}}$ is a (multiplicative) group isomorphic to the additive group $\mathbb{F}_{q^{m}}$ and comprise the characters $\left\{\chi_{c} \mid c \in \mathbb{F}_{q^{m}}\right\}$. The identity of such group is the trivial additive character $\chi_{0}$. We introduce a useful notation.

Definition 3.1. Fix $n$ a positive integer, $d$ a divisor of $n$ and $a \in \mathbb{F}_{q^{d}}$. Let $I_{n, d, a}$ be the characteristic function for elements in $\mathbb{F}_{q^{n}}$ with trace $a$ over $\mathbb{F}_{q^{d}}$, and let $\Omega_{n}$ be the characteristic function for primitive elements in $\mathbb{F}_{q^{n}}$, i.e., for $\alpha \in \mathbb{F}_{q^{n}}$ we have that

$$
I_{n, d, a}(\alpha)=\left\{\begin{array}{ll}
1 & \text { if } \operatorname{Tr}_{n / d}(\alpha)=a, \\
0 & \text { otherwise }
\end{array} \quad \text { and } \quad \Omega_{n}(\alpha)= \begin{cases}1 & \text { if } \alpha \text { is primitive } \\
0 & \text { otherwise }\end{cases}\right.
$$

In addition, set $\theta(q)=\frac{\varphi\left(q^{n}-1\right)}{q^{n}-1}$.

The following results provide expressions for the functions $\Omega_{n}$ and $I_{n, d, a}$ by means of characters.

Lemma 3.2. For every $\beta \in \mathbb{F}_{q^{n}}$, we have that

$$
\Omega_{n}(\beta)=\theta(q) \sum_{t \mid q^{n}-1} \frac{\mu(t)}{\varphi(t)} \sum_{\eta \in \Gamma(t)} \eta(\beta),
$$

where $\mu$ is the Möbius function over the integers.

For the proof of the previous lemma, see Theorem 2.8 of [5] and the comments thereafter.

Lemma 3.3. Let $m$ be a divisor of $n$ and $\gamma \in \mathbb{F}_{q^{n}}$ such that $\operatorname{Tr}_{n / m}(\gamma)=$ $a \in \mathbb{F}_{q^{m}}$. If $\chi$ denotes the canonical additive character of $\mathbb{F}_{q^{n}}$, then for any $\beta \in \mathbb{F}_{q^{n}}$ we have that

$$
I_{n, d, a}(\beta)=\frac{1}{q^{m}} \sum_{c \in \mathbb{F}_{q^{m}}} \chi_{c}(\beta-\gamma)=\frac{1}{q^{m}} \sum_{c \in \mathbb{F}_{q} m} \chi_{c}(\beta) \chi_{c}(\gamma)^{-1}
$$

For the proof of the previous lemma, see Subsection 2.3.1 of [5].

3.2. Auxiliary lemmas From Corollary 1.2 in [8], we have the following result.

LEMma 3.4. Let $n>1$ be an integer that is not a prime power, $1<$ $k<\sigma_{0}(n)$, and let $\mathbf{d}=\left(d_{1}, \ldots, d_{k}\right) \in \Lambda_{k}(n)$. Then the number of $k$-tuples $\left(x_{1}, \ldots, x_{k}\right) \in \mathbb{F}(\mathbf{d})$ such that $x_{1}+\cdots+x_{k}=0$ equals

$$
q^{d_{1}+\cdots+d_{k}-\lambda(\mathbf{d})} .
$$


We further require effective upper bounds on the functions $W$ and $\lambda(\mathbf{d})$. We have the following results.

LEMma 3.5. (i) If $W(t)$ is the number of squarefree divisors of $t$, then for all $t \geq 3$,

$$
W(t-1)<t^{\frac{0.96}{\log \log t}} .
$$

(ii) If $n>1$ is an integer that is not a prime power, $1<k<\sigma_{0}(n)$ and $\mathbf{d} \in \Lambda_{k}(n)$, then

$$
\lambda(\mathbf{d}) \leq n-\varphi(n),
$$

where $\varphi(n)$ is the Euler Totient Function.

Proof. Item (i) is a straightforward consequence of inequality (4.1) in [3]. For item (ii) observe that, as stated in Remark 2, $\lambda(\mathbf{d})$ equals the degree of the least common multiple of the polynomials $x^{d_{1}}-1, \ldots, x^{d_{k}}-1$. Since $d_{1}<\cdots<d_{k}<n$, if $p_{1}, \ldots, p_{t}$ are the distinct prime divisors of $n$, we have that the polynomials $x^{d_{1}}-1, \ldots, x^{d_{k}}-1$ divide the polynomial

$$
\operatorname{lcm}\left(x^{n / p_{1}}-1, \ldots, x^{n / p_{t}}-1\right) .
$$

An inclusion-exclusion argument shows that the previous polynomial has degree $n-\varphi(n)$ and the result follows.

Lemma 3.6 (see Lemma 4.1 of [5]). If a is a positive integer and $p_{1}, \ldots, p_{j}$ are the distinct prime divisors of $t$ such that $p_{i} \leq 2^{a}$, then

$$
W(t) \leq c_{t, a} t^{1 / a}, \quad \text { where } \quad c_{t, a}:=\frac{2^{j}}{\left(p_{1} \ldots p_{j}\right)^{1 / a}}
$$

In particular,

$$
c_{t, 4}<\left\{\begin{array}{ll}
4.9 & \text { for } t \text { even, } \\
2.9 & \text { for } t \text { odd },
\end{array} \quad \text { and } \quad c_{t, 8}<4514.7 .\right.
$$

\section{Proof of the main result}

Let $N(n, \mathbf{d}, \mathbf{a})$ be the number of primitive elements $\alpha \in \mathbb{F}_{q^{n}}$ such that $\operatorname{Tr}_{n / d_{i}}(\alpha)=a_{i}$. In particular,

$$
N(n, \mathbf{d}, \mathbf{a})=\sum_{w \in \mathbb{F}_{q^{n}}} \Omega_{n}(\omega) \cdot \prod_{i=1}^{k} I_{n, d_{i}, a_{i}}(w) .
$$

Since the $k$-tuple $\left(a_{1}, \ldots, a_{k}\right)$ is $\mathbf{d}$-admissible, we have seen that there exists $\beta \in \mathbb{F}_{q^{n}}$ such that $\operatorname{Tr}_{\frac{n}{t} / d_{i}}(\beta)=a_{i}$ for $1 \leq i \leq k$. Write $D=d_{1}+\cdots+d_{k}$ 
and, for a generic $\mathbf{c}=\left(c_{1}, \ldots, c_{k}\right) \in \mathbb{F}(\mathbf{d})$, write $s(\mathbf{c})=\sum_{i=1}^{k} c_{i}$. From Lemmas 3.2 and 3.3, we have that

$$
\begin{aligned}
\frac{q^{D} N(n, \mathbf{d}, \mathbf{a})}{\theta(q)} & =\sum_{w \in \mathbb{F}_{q^{n}}} \sum_{t \mid q^{n}-1} \frac{\mu(t)}{\varphi(t)} \sum_{\eta \in \Gamma(t)} \eta(w) \cdot \prod_{i=1}^{k}\left(\sum_{c_{i} \in \mathbb{F}_{q^{d}} d_{i}} \chi_{c_{i}}(w) \cdot \chi_{c_{i}}(\beta)^{-1}\right) \\
& =\sum_{w \in \mathbb{F}_{q^{n}}} \sum_{\mathbf{c} \in \mathbb{F}(\mathbf{d})} \sum_{t \mid q^{n}-1} \frac{\mu(t)}{\varphi(t)} \sum_{\eta \in \Gamma(t)} \eta(w) \cdot \chi_{s(\mathbf{c})}(w) \cdot \chi_{s(\mathbf{c})}(-\beta) \\
& =\sum_{\mathbf{c} \in \mathbb{F}(\mathbf{d})} \sum_{t \mid q^{n}-1} \frac{\mu(t)}{\varphi(t)} \sum_{\eta \in \Gamma(t)} \chi_{s(\mathbf{c})}(-\beta) \cdot G_{n}\left(\eta, \chi_{s(\mathbf{c})}\right),
\end{aligned}
$$

where $G_{n}\left(\eta, \chi_{s(\mathbf{c})}\right)=\sum_{w \in \mathbb{F}_{q} n} \eta(w) \cdot \chi_{s(\mathbf{c})}(w)$ denotes a Gauss sum. We use the orthogonality relations to obtain

$$
G_{n}\left(\eta, \chi_{s(\mathbf{c})}\right)= \begin{cases}q^{n} & \text { if } \eta \in \Gamma(1) \text { and } s(\mathbf{c})=0, \\ 0 & \text { if } \eta \in \Gamma(1) \text { and } s(\mathbf{c}) \neq 0, \\ 0 & \text { if } \eta \notin \Gamma(1) \text { and } s(\mathbf{c})=0,\end{cases}
$$

and in the remaining cases we have the well-known identity $\left|G_{n}\left(\eta, \chi_{s(\mathbf{c})}\right)\right|=$ $q^{n / 2}$. In addition, if $s(\mathbf{c})=0$, then $\chi_{s(\mathbf{c})}(-\beta)=\chi_{0}(-\beta)=1$. In particular, we may rewrite

$$
\frac{q^{D} N(n, \mathbf{d}, \mathbf{a})}{\theta(q)}=\sum_{\substack{\mathbf{c} \in \mathbb{F}(\mathbf{d}) \\ s(\mathbf{c})=0}} q^{n}+\underbrace{\sum_{\substack{\mathbf{c} \in \mathbb{F}(\mathbf{d}) \\ s(\mathbf{c}) \neq 0}} \sum_{\substack{t \mid q^{n}-1 \\ t \neq 1}} \frac{\mu(t)}{\varphi(t)} \sum_{\eta \in \Gamma(t)} \chi_{s(\mathbf{c})}(-\beta) \cdot G_{n}\left(\eta, \chi_{s(\mathbf{c})}\right)}_{S} .
$$

From Lemma 3.4, we obtain the following equality

$$
\frac{q^{D} N(n, \mathbf{d}, \mathbf{a})}{\theta(q)}=q^{n+D-\lambda(\mathbf{d})}+S
$$

We observe that $\left|\chi_{s(\mathbf{c})}(-\beta)\right|=1$ and $\left|G_{n}\left(\eta, \chi_{s(\mathbf{c})}\right)\right|=q^{n / 2}$ in every term of the sum $S$. Recall that there exist exactly $\varphi(t)$ elements in $\Gamma(t)$ and the function $\mu$ has absolute value 1 at squarefree integers, and vanishes everywhere else. In particular, we obtain the following inequality

$$
|S|<\sum_{\substack{\mathbf{c} \in \mathbb{F}(\mathbf{d}) \\ s(\mathbf{c}) \neq 0}} \sum_{\substack{t \mid q^{n}-1 \\ t \text { squarefree }}} q^{n / 2}=q^{n / 2+D} \cdot W\left(q^{n}-1\right) .
$$

Hence,

$$
\frac{q^{D} N(n, \mathbf{d}, \mathbf{a})}{\theta(q)}>q^{n+D-\lambda(\mathbf{d})}-q^{n / 2+D} \cdot W\left(q^{n}-1\right) \geq 0,
$$

provided that $q^{n / 2-\lambda(\mathbf{d})} \geq W\left(q^{n}-1\right)$. 
4.1. Proof of Theorem 2.4 Set $\operatorname{lcm}\left(d_{1}, \ldots, d_{k}\right)=\frac{n}{t}$, where $t>1$ is a divisor of $n$. Since there do not exist $1 \leq i, j \leq k$ such that $d_{i}$ divides $d_{j}$, we have that $d_{i}<n / t$ for any $1 \leq i \leq k$ and then, from Lemma 3.5, we have that $\lambda(\mathbf{d}) \leq n / t-\varphi(n / t)$. From hypothesis the $k$-tuple $\left(a_{1}, \ldots, a_{k}\right)$ is $\mathbf{d}$ admissible and then, as stated in Remark 2, Theorem 4.1 of [8] implies that there exist $q^{n / t-\lambda(\mathbf{d})} \geq q^{\varphi(n / t)}>1$ elements $\theta \in \mathbb{F}_{q^{n / t}}$ such that $\operatorname{Tr}_{\frac{n}{t}} / d_{i}(\theta)=$ $a_{i}$ for $1 \leq i \leq k$. In particular, there exists $\theta_{0} \in \mathbb{F}_{q^{t}}$ with such a property in a way that $\theta_{0} \neq 0$. Since $\theta_{0} \neq 0$, Theorem 1.1 implies that there exists a primitive element $\alpha \in \mathbb{F}_{q^{n}}$ such that $\operatorname{Tr}_{n / \frac{n}{t}}(\alpha)=\theta_{0}$ and then, by the transitivity of the trace, we have that

$$
\operatorname{Tr}_{n / d_{i}}(\alpha)=\operatorname{Tr}_{\frac{n}{t} / d_{i}}\left(\theta_{0}\right)=a_{i}, 1 \leq i \leq k .
$$

\section{Theorem 2.3 under the condition $\operatorname{gcd}\left(d_{i}, d_{j}\right)=1$}

In this section, we discuss the existence of primitive elements of $\mathbb{F}_{q^{n}}$ with arbitrary prescribed traces over extensions $\mathbb{F}_{q^{d_{i}}}$ under the following condition:

$$
\operatorname{gcd}\left(d_{i}, d_{j}\right)=1 \text { for } 1 \leq i<j \leq k \text {. }
$$

We observe that the former condition is not restrictive when $k=2$. In fact, if $d_{1}<d_{2}$ are divisors of $n$ and $d=\operatorname{gcd}\left(d_{1}, d_{2}\right)$, we have that $\mathbb{F}_{q^{d_{i}}}=\mathbb{F}_{Q^{e_{i}}}$ where $e_{i}=d_{i} / d$ satisfy $\operatorname{gcd}\left(e_{1}, e_{2}\right)=1$. We obtain asymptotic and concrete results, that are displayed in the following theorem.

TheOREM 5.1. Let $n>1$ be an integer that is not a prime power, $1<$ $k<\sigma_{0}(n)$ and $\mathbf{d}=\left(d_{1}, \ldots, d_{k}\right) \in \Lambda_{k}(n)$ be such that $\operatorname{gcd}\left(d_{i}, d_{j}\right)=1$ for every $1 \leq i<j \leq k$. Furthermore, let $\mathbf{a}=\left(a_{1}, \ldots, a_{k}\right) \in \mathbb{F}(\mathbf{d})$ be a $\mathbf{d}$-admissible $k$-tuple. Then there exists a primitive element $\alpha \in \mathbb{F}_{q^{n}}$ with prescribed traces $\operatorname{Tr}_{n / d_{i}}(\alpha)=a_{i}$ provided that one of the following holds:

(a) $k \geq 3$;

(b) $k=2$ and

(b.1) $d_{1} \geq 5$ and $q \geq 5$ if $\left(d_{1}, d_{2}\right)=(5,6)$;

(b.2) $d_{1}=4$ and either $d_{2} \geq 11$, or $d_{2} \geq 9$ and $q \geq 3$, or $d_{2}=5,7$ and $q \geq e^{e^{6.7}}$

(b.3) $d_{1}=3$ and either $d_{2} \geq 38$, or $d_{2} \geq 5$ and $q \geq e^{e^{26.1}}$.

Proof. We may assume that $\operatorname{lcm}\left(d_{1}, \ldots, d_{k}\right)=n$ since otherwise the result is directly implied by Theorem 2.4. For $k \geq 2$, the condition $\operatorname{gcd}\left(d_{i}, d_{j}\right)=$ 1 implies that

$$
\lambda(\mathbf{d})=d_{1}+\cdots+d_{k}-k+1
$$


From Lemma 3.6, Theorem 2.3 and the equation above, it suffices to verify that

$$
\frac{n}{2}-\sum_{i=1}^{k} d_{i}+k-1 \geq \frac{n}{a}+\log _{q}\left(c_{q^{n}, a}\right),
$$

for some $a \geq 3$. We always take $a=4$ or $a=8$. Since $\operatorname{lcm}\left(d_{1}, \ldots, d_{k}\right)=n$, $2 \leq d_{1}<\cdots<d_{k}$ and $\operatorname{gcd}\left(d_{i}, d_{j}\right)=1$, we have that

$$
d_{1} \ldots d_{k}=n .
$$

We provide the proof of items (a) and (b) separately.

5.1. The case $k \geq 3$ We split the proof into cases.

(i) $k \geq 4$ : Let $2=p_{1}<p_{2}<\cdots$ be the increasing sequence of the prime numbers. We have that $p_{\ell} \leq d_{\ell}$ and then

$$
p_{\ell} \leq d_{\ell} \leq\left(\frac{n}{p_{1} \ldots p_{\ell-1}}\right)^{\frac{1}{k+1-\ell}},
$$

for $1 \leq \ell \leq k$, where the empty product equals 1 . Furthermore,

$$
\frac{n}{2}-\left[\log _{7}\left(\frac{n}{30}\right)+2\right] \sqrt{\frac{n}{2}}-\frac{n}{30}+3 \geq \frac{n}{4}+2
$$

for $n \geq 39$. Since $\left(\frac{n}{p_{1} \ldots p_{\ell-1}}\right)^{\frac{1}{k+1-\ell}}<\sqrt{\frac{n}{2}}$ for every $1 \leq \ell \leq k-1$ and $n \geq 2 \cdot 3 \cdot 5 \cdot 7^{k-3} \geq 210$, the LHS of inequality (5) is greater than the LHS of inequality (7). Taking $a=4$, the RHS of inequality (7) is greater than the RHS of inequality (5). This concludes the case $k \geq 4$.

(ii) $k=3$ : In the same way, we have that the following inequality

$$
\frac{n}{2}-\sqrt[3]{n}-\sqrt{\frac{n}{2}}-\frac{n}{6}+2 \geq \frac{n}{8}+12.2
$$

holds true for $n \geq 107$. Since the LHS of inequality (5) is greater than the LHS of inequality (8), and the RHS of inequality (8) is greater than the RHS of inequality (5) with $a=8$, we are done unless $n \in\{30=$ $2 \cdot 3 \cdot 5,42=2 \cdot 3 \cdot 7,60=3 \cdot 4 \cdot 5,66=2 \cdot 3 \cdot 11,70=2 \cdot 5 \cdot 7,78=$ $2 \cdot 3 \cdot 13,84=3 \cdot 4 \cdot 7,90=2 \cdot 5 \cdot 9,102=2 \cdot 3 \cdot 17,105=3 \cdot 5 \cdot 7\}$, which are the numbers smaller than 107 that split into at least three non-trivial relatively prime factors. We now consider the cases:

(ii.1) $\left(d_{1}, d_{2}\right)=(3,5)$ : If $n \geq 60$ then $\frac{n}{2}-3-5-\frac{n}{15}+2 \geq \frac{n}{4}+2$, and we argue as above with $a=4$.

(ii.2) $\left(d_{1}, d_{2}\right)=(3,4)$ : If $n \geq 42$ then $\frac{n}{2}-3-4-\frac{n}{12}+2 \geq \frac{n}{4}+2$, and we argue as above with $a=4$. 
(ii.3) $\left(d_{1}, d_{2}\right)=(2,5)$ : If $n \geq 47$ then $\frac{n}{2}-2-5-\frac{n}{10}+2 \geq \frac{n}{4}+2$, and we argue as above with $a=4$.

(ii.4) $\left(d_{1}, d_{2}\right)=(2,3)$ : If $n \geq 60$ then $\frac{n}{2}-2-3-\frac{n}{6}+2 \geq \frac{n}{4}+2$, and we argue as above with $a=4$. Hence, there only remains $n \in\{30,42\}$.

For $n=30$, the inequality

$$
\frac{30}{2}-2-3-5+2 \geq \frac{30}{8}+\log _{q}(4514.7)
$$

holds true if $q \geq 14$ and we argue as above with $a=8$. Therefore there only remains the cases $q \in\{2,3,4,5,7,8,9,11,13\}$, for which the inequality $q^{n / 2-\lambda(\mathbf{d})} \geq W\left(q^{n}-1\right)$ can be directly verified.

For $n=42$, the inequality

$$
\frac{42}{2}-2-3-7+2 \geq \frac{42}{8}+\log _{q}(4514.7)
$$

holds true if $q \geq 5$ and we argue as above with $a=8$. Therefore it only remains the cases $q \in\{2,3,4\}$, for which the inequality $q^{n / 2-\lambda(\mathbf{d})} \geq W\left(q^{n}-1\right)$ can be directly verified.

5.2. The case $k=2$ As in previous cases, it suffices to prove that

$$
\frac{n}{2}-d_{1}-d_{2}+1 \geq \frac{n}{a}+\log _{q}\left(c_{q^{n}, a}\right),
$$

for $a \in\{4,8\}$. Notice that if $d_{1} \geq 8$ then $d_{2} \geq 9$ and $\left(d_{1}-4\right)\left(d_{2}-4\right) \geq 20 \geq$ $12+4 \log _{q}\left(c_{q^{n}, 4}\right)$. Therefore, inequality (9) holds true with $a=4$. Table 1 provides the ranges of $q, d_{1}, d_{2}$ where inequality (9) holds and the value of $a$ that is used.

Table 1 compiles exceptions $\left(q, d_{1}, d_{2}\right)$ for inequality (9), assuming that either $d_{1} \geq 5$, or $d_{1}=4$ and $d_{2} \geq 7$, or $d_{1}=3$ and $d_{2} \geq 38$. With respect to these ranges, the exceptional triples have reasonably small parameters. Using the software SageMath we verify that such triples $\left(q, d_{1}, d_{2}\right)$ satisfy inequality (3), with the exception of $\left(q, d_{1}, d_{2}\right)=(q, 5,6)$ with $q<5$ and $\left(q, d_{1}, d_{2}\right)=(2,4,9)$.

For $\left(q, d_{1}, d_{2}\right)=(q, 3,4)$, inequality (3) does not hold for any prime power $q$. For the remaining cases, that is, $\left(q, d_{1}, d_{2}\right)=(q, 4,5)$ and $\left(q, d_{1}, d_{2}\right)=$ $\left(q, 3, d_{2}\right)$ with $5 \leq d_{2} \leq 37$, Lemma $3.5(\mathrm{i})$ (or inequality (9) with $a=8$ provided $d_{1}=3$ and $\left.17 \leq d_{2} \leq 37\right)$ ensures that there exists a computable constant $q_{0}$ depending only on $d_{1}$ and $d_{2}$ such that inequality (3) holds for every $q \geq q_{0}$. 


\begin{tabular}{|c|c|c|c|}
\hline$d_{1}$ & $d_{2}$ & $q$ & $a$ \\
\hline \hline 7 & $\geq 11$ & $\forall q$ & 4 \\
\hline 7 & 10 & $\geq 5$ & 4 \\
\hline 7 & 9 & $\geq 8$ & 4 \\
\hline 7 & 8 & $\geq 37$ & 8 \\
\hline 6 & $\geq 15$ & $\forall q$ & 4 \\
\hline 6 & 13 & $\geq 3$ & 4 \\
\hline 6 & 11 & $\geq 3$ & 8 \\
\hline 6 & 7 & $\geq 11$ & 8 \\
\hline 5 & $\geq 19$ & $\forall q$ & 8 \\
\hline 5 & $\geq 14$ & $\geq 3$ & 8 \\
\hline 5 & $\geq 12$ & $\geq 4$ & 8 \\
\hline 5 & 11 & $\geq 5$ & 8 \\
\hline 5 & 9 & $\geq 9$ & 8 \\
\hline 5 & 8 & $\geq 17$ & 8 \\
\hline 5 & 7 & $\geq 53$ & 8 \\
\hline 5 & 6 & $\geq 839$ & 8 \\
\hline 4 & $\geq 31$ & $\forall q$ & 8 \\
\hline 4 & $\geq 23$ & $\geq 3$ & 8 \\
\hline
\end{tabular}

\begin{tabular}{|c|c|c|c|}
\hline$d_{1}$ & $d_{2}$ & $q$ & $a$ \\
\hline \hline 4 & $\geq 19$ & $\geq 4$ & 8 \\
\hline 4 & 17 & $\geq 5$ & 8 \\
\hline 4 & 15 & $\geq 7$ & 8 \\
\hline 4 & 13 & $\geq 13$ & 8 \\
\hline 4 & 11 & $\geq 29$ & 8 \\
\hline 4 & 9 & $\geq 274$ & 8 \\
\hline 4 & 7 & $\geq 2.039 \cdot 10^{7}$ & 8 \\
\hline 3 & $\geq 114$ & $\forall q$ & 8 \\
\hline 3 & $\geq 78$ & $\geq 3$ & 8 \\
\hline 3 & $\geq 65$ & $\geq 4$ & 8 \\
\hline 3 & $\geq 58$ & $\geq 5$ & 8 \\
\hline 3 & $\geq 52$ & $\geq 7$ & 8 \\
\hline 3 & $\geq 49$ & $\geq 8$ & 8 \\
\hline 3 & 47 & $\geq 9$ & 8 \\
\hline 3 & 46 & $\geq 11$ & 8 \\
\hline 3 & $\geq 43$ & $\geq 13$ & 8 \\
\hline 3 & $\geq 40$ & $\geq 17$ & 8 \\
\hline 3 & 38 & $\geq 23$ & 8 \\
\hline
\end{tabular}

TABLE 1. Ranges of $q, d_{1}$ and $d_{2}$ where inequality (9) holds true and the value of $a$ that is used.

REMARK. For $k=2, d_{1}=2$ and $n=\operatorname{lcm}\left(2, d_{2}\right)$, we have that $q^{n / 2-\lambda(\mathbf{d})}<$ $1<W\left(q^{n}-1\right)$, and so Theorem 2.3 is inconclusive. Moreover, in this setting we can actually provide genuine exceptions. In fact, let $n=2 \cdot N, N>1$ odd and choose $b \in \mathbb{F}_{q^{2}}$ in way that $\operatorname{Tr}_{N / 1}(b)=0$. In particular, the pair $(b, 0) \in \mathbb{F}_{q^{2}} \times \mathbb{F}_{q^{N}}$ is $(2, N)$-admissible. However, there is no primitive element $\alpha \in \mathbb{F}_{q^{n}}$ with zero trace over $\mathbb{F}_{q^{N}}$. In fact, any element $\alpha \in \mathbb{F}_{q^{n}}$ with zero trace over $\mathbb{F}_{q^{N}}$ satisfies $\alpha^{q^{N}}=-\alpha$ and, consequently, $\alpha^{2\left(q^{N}-1\right)}=1$. But such an $\alpha$ cannot be primitive since $2\left(q^{N}-1\right)<q^{n}-1$ for every $q \geq 2$.

\section{Conclusion}

In this paper we have discussed the existence of primitive elements of finite fields with prescribed traces in intermediate extensions. Our main result provides a sufficient condition for the existence of such elements. This condition is encoded in an inequality, which is further explored in order to obtain concrete results on the existence of these elements; this is presented in Theorem 5.1 .

It would be desirable to explore the validity of Theorem 5.1 without the restriction $\operatorname{gcd}\left(d_{i}, d_{j}\right)=1$ or at least complete this theorem, exploring 
the remaining cases under this restriction. For instance, by using a sieving method that is traditional in this kind of problem (see [2,3]), one can remove the restrictions $q \geq 5$ and $q \geq 3$ in items (b.1) and (b.2) of Theorem 5.1. Within the approach of this paper, we believe that any such improvement would have to go through sharper estimates on the character sums appearing in the proof of Theorem 2.3.

\section{Acknowledgement}

We thank the anonymous referee for the suggestions that substantially improved the presentation of this work.

\section{References}

[1] S. D. Cohen. Primitive elements and polynomials with arbitrary trace. Discrete Math., 83(1):1-7, 1990.

[2] S. D. Cohen and S. Huczynska. The primitive normal basis theorem - without a computer. J. London Math. Soc., 67(1):41-56, 2003.

[3] S. D. Cohen., T. Oliveira e Silva and T. Trudgian. On consecutive primitive elements in a finite field. Bull. Lond. Math. Soc. 47(3): 418-426, 2015.

[4] W. Diffie and M. Hellman. New directions in cryptography. IEEE Trans. Information Theory, 22(6):644-654, 1976.

[5] G. Kapetanakis and L. Reis. Variations of the Primitive Normal Basis Theorem. Des. Codes Cryptogr. 87(7): 1459-1480, 2019.

[6] H. W. Lenstra, Jr and R. J. Schoof. Primitive normal bases for finite fields. Math. Comp., 48(177):217-231, 1987.

[7] R. Lidl and H. Niederreiter. Finite Fields, volume 20 of Encyclopedia of Mathematics and its Applications. Cambridge University Press, Cambridge, second edition, 1997.

[8] L. Reis. Counting solutions of special linear equations over finite fields. Finite Fields Appl. 68: 101759, 2020.

Departamento de Matemática, Universidade Federal de Minas Gerais (UFMG), Belo Horizonte, MG, 30270-901, Brazil.

e-mail: lucasreismat@ufmg.br

Departamento de Matemática, Universidade Federal de Ouro Preto (UFOP),

Ouro Preto, MG, 35400-000, Brazil.

e-mail: savio.ribas@ufop.edu.br 